\title{
Planned observations of thermal plasma drifts and solar wind interactions in the Martian ionosphere
}

\author{
A. W. Yau ${ }^{1,2}$, G. P. Garbe ${ }^{1}$, M. J. Greffen ${ }^{1}$, W. Miyake ${ }^{3}$, and E. Sagawa ${ }^{4}$ \\ ${ }^{1}$ Department of Physics and Astronomy, University of Calgary, Calgary, Alberta, Canada \\ ${ }^{2}$ Herzberg Institute of Astrophysics, Victoria, British Columbia, Canada \\ ${ }^{3}$ Communications Research Laboratory, Koganei, Tokyo 184, Japan \\ ${ }^{4}$ Hiraiso Solar Terrestrial Research Center, CRL, Nakaminato, Japan
}

(Received August 1, 1997; Revised November 26, 1997; Accepted December 2, 1997)

\begin{abstract}
The structure and dynamics of the Martian ionosphere are believed to be strongly dependent on the nature, magnitude, and topology of its magnetic field, and whether or not Mars has an intrinsic magnetic field. Due to the weak magnetic field on Mars, the Martian ionosphere interacts directly with the solar wind, resulting in significant outflows of keV ion beams and lower-energy "pick-up" ions. We discuss the planned study of ion drifts and solar wind interactions in the Martian ionosphere using the Planet-B Thermal Plasma Analyzer.
\end{abstract}

\section{Introduction}

In this letter, we discuss the study of the structure and dynamics of the Martian ionosphere and its interaction with the solar wind in light of planned observations using the Planet-B Thermal Plasma Analyzer (TPA) instrument. The absence of a strong magnetic field on Mars results in direct interactions of the thermal plasma in the upper atmosphere with the solar wind. The question of whether or not Mars has an intrinsic magnetic field remains controversial (Russell, 1986; Dolginov, 1986) and will be the primary scientific focus of the Planet-B mission. The presence of an intrinsic field, however weak, is believed to have direct consequences on the structure and dynamics of the Martian ionosphere.

Previous observations suggest the presence of two or perhaps three distinct boundaries in the Martian magnetosphere (Axford, 1991): an outer boundary delimiting the bulk solar wind flow and magnetohydrodynamic waves outside and the decrease in electron plasma temperature inside, a second boundary marking the transition from a region of external plasma to one dominated by tailward flowing ionospheric ions, and possibly a third boundary encompassing a region of intrinsic magnetic field.

\section{Thermal Plasma Analyzer}

The Planet-B spacecraft is scheduled for launch from Kagoshima Space Center, Japan, in July 1998, and will orbit around Mars in a highly elliptic orbit (150 km periapsis altitude, 15 Martian radii apoapsis distance, $137.6^{\circ}$ inclination) upon its arrival in December 1999. The Thermal Plasma Analyzer (TPA) is one of 15 scientific instruments on Planet-B; see Yamamoto et al. (1998, this issue) for an overview of the Planet-B mission and instrument payload. The TPA is designed to measure the drift velocity, temperature, and composition of thermal-energy $(1-20 \mathrm{eV})$ ions in the Martian

Copy right $(\mathrm{C})$ The Society of Geomagnetism and Earth, Planetary and Space Sciences (SGEPSS); The Seismological Society of Japan; The Volcanological Society of Japan; The Geodetic Society of Japan; The Japanese Society for Planetary Sciences. topside ionosphere in its thermal ion mode, and the energy, pitch angle and angular distributions of suprathermal (10 $200 \mathrm{eV}$ ) ions or electrons in the Martian magnetosphere and magnetotail in its suprathermal mode.

The TPA sensor is mounted on a boom along the spacecraft spin plane. Figure 1 is a schematic cross section of the sensor through its axis of rotational symmetry. The sensor contains four entrance slits around the sensor circumference, allowing particle entry in a plane perpendicular to the spacecraft spin plane. The entrance slits are located at $1 \mathrm{~m}$ from the spacecraft sidepanel. The sensor exterior surface can be biased either positive or negative with respect to the spacecraft, to partially compensate for the spacecraft potential. Inside the entrance aperture slits, the sensor contains an electrostatic deflection gate composed of a pair of time-of-flight (TOF) electrodes, a hemispherical electrostatic analyzer (HEA), and an imaging microchannel plate (MCP) detector. The inner hemisphere of the HEA can be biased either positive or negative relative to the outer hemisphere for electron or ion measurements, respectively. The HEA disperses incident particles in energy per charge and azimuth (in the plane perpendicular to the sensor axis of symmetry) onto its hemispheric plane. Its ion optics is essentially identical to that in the Freja Cold Plasma Analyzer (Whalen et al., 1994). Particles at a fixed energy-per-charge and incident azimuth and different elevation angles and entrance positions are focussed onto a point in the plane, at a radial distance from the center of the plane that increases with the energy-per-charge. The maximum and minimum energy-per-charge, $E_{\max }$ and $E_{\min }$, of the measured particles are defined by the electrostatic voltages at the entrance aperture (VEA), the outer and inner hemispheres (VES and VSA, respectively) of the hemispherical electrostatic analyzer, and the guard grid (VGD) above the MCP. $E_{\max } / E_{\min }$ is typically about 10 . In ion measurements, the pulse timing and duration of the electrode potentials in the TOF gate pre-select the mass range of particles entering the HEA. 


\section{TPA Simulated Ion Trajectories}
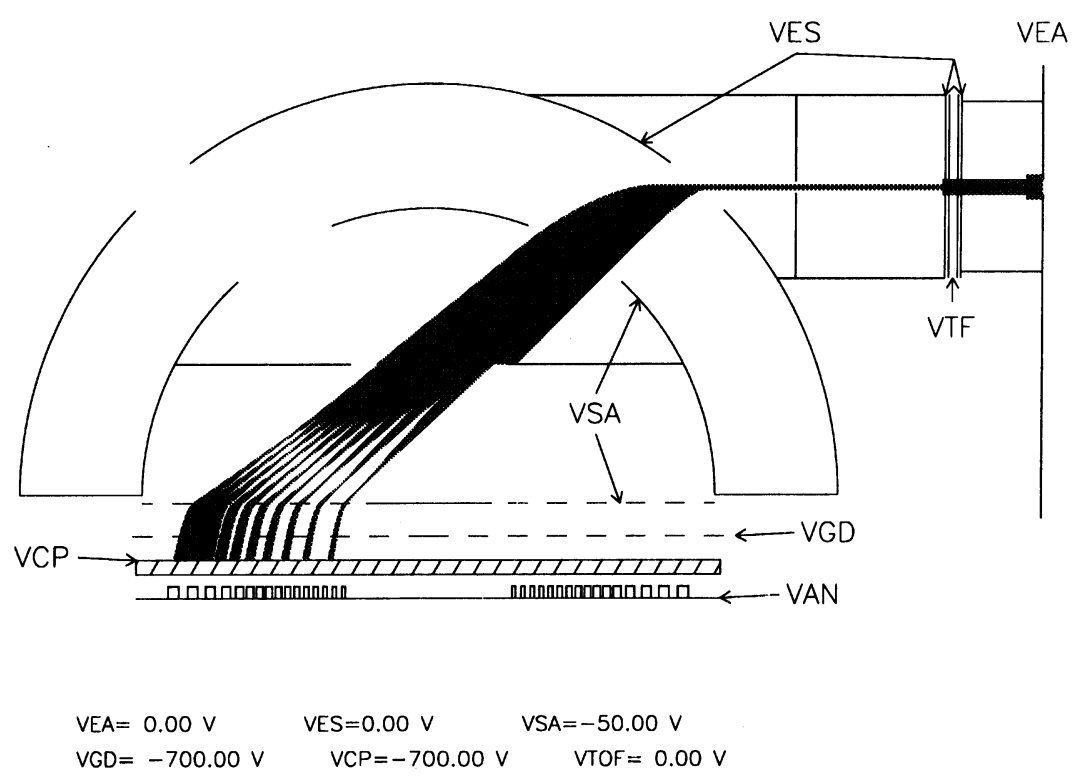

Fig. 1. Schematic cross section of Thermal Plasma Analyzer (TPA) sensor through its axis of rotational symmetry depicting the pixel locations on the anode and the simulated trajectories of $0.5-10 \mathrm{eV} / \mathrm{q}$ ions inside the sensor. The entrance aperture and time of flight gate on the left side of the figure are omitted for the sake of clarity.

Particles arriving at the MCP produce charges in it which are then proximity focussed and collected onto a discrete anode. The anode contains 256 pixel elements in 16 concentric rows and 16 radial columns. Two of the 16 pixel columns (columns 1 and 9) are aligned parallel to the spacecraft spin axis, while two other pixel columns (columns 5 and 13) are perpendicular to it. Figure 1 depicts the pixel rows on the anode and the simulated trajectories of $0.5-10 \mathrm{eV}$ ions inside the sensor for $\mathrm{VSA}=-50 \mathrm{~V}$. (VEA $=\mathrm{VES}=0 \mathrm{~V}$ and $\mathrm{VGD}=\mathrm{VCP}=-700 \mathrm{~V} ; \mathrm{VCP}$ and VAN are the potentials at the front of the MCP and the anode, respectively.) The sensor uses a 16 by 16 preamplifier coincident circuit to detect charge build-up ("hit") at each pixel and to determine the pixel location of the detected charge pulse. In addition, it clocks the arrival time of the pulse relative to the TOF gate opening using 256 time-of-flight bins. The measured pixel column defines the azimuth of the incident particle perpendicular to the spacecraft spin plane. The measured pixel row and time-of-flight bin combine to define the particle energyper-charge and mass-per-charge. The instrument has a maximum throughput of about $3 \times 10^{5}$ particle hits per second. During a measurement interval, the pixel row, column, and time-of-flight bin address of each hit is stored in memory. At the end of the interval, the stored address data are processed to construct a 2-dimensional energy-azimuthal distribution (image) of ion counts for each selected ion species.

In the thermal ion mode, successive "images" over half a spin are combined to derive the ion velocity, temperature, and density of the selected species, as well as the temporal variation of the spin axis component of the ion velocity during the half-spin (using data from pixel columns 1 and 9). VSA steps between two values (nominally $-50 \mathrm{~V}$ and $-100 \mathrm{~V}$ ) every other spin to provide sampling at 32 energy steps between $\sim 1$ and $20 \mathrm{eV}$ over a 2 -spin period. In the suprathermal modes, measurements over half a spin are used to derive detailed energy, pitch-angle, and azimuthal distributions of electrons or ions up to $200 \mathrm{eV}$; VSA steps between its maximum and half-maximum values every other spin $(-1000 \mathrm{~V}$ and $-500 \mathrm{~V}$ in the ion mode and $+1000 \mathrm{~V}$ and $+500 \mathrm{~V}$ in the electron mode).

\section{Plasma Scale Height and Drift}

From retarding potential analyzer (RPA) measurements on the Viking 1 and 2 landers near solar minimum, Hanson et al. (1977) inferred the presence of $\mathrm{O}_{2}^{+}$and $\mathrm{CO}_{2}^{+}$ions in the Martian ionosphere, with a peak ion density of $\sim 10^{5} \mathrm{~cm}^{-3}$ and an $\mathrm{O}_{2}^{+} / \mathrm{CO}_{2}^{+}$ratio of $\sim 9$ at $130 \mathrm{~km}$ altitude. At higher altitudes, $\mathrm{O}^{+}$was also present, and had a peak density of $<10^{3} \mathrm{~cm}^{-3}$ at $225 \mathrm{~km}$. Both Viking 1 and 2 measurements were made at $44^{\circ}$ solar zenith angle (SZA), and were in reasonable accord with earlier occultation measurements. On Viking 1, the measured plasma density scale height was 29 $\mathrm{km}$ up to $350 \mathrm{~km}$. The ion temperature increased by more than a decade from $150^{\circ} \mathrm{K}$ at $130 \mathrm{~km}$ to $\sim 2500^{\circ} \mathrm{K}$ at $300 \mathrm{~km}$. On Viking 2, both the density and temperature were more structured than on Viking 1. The density dropped rapidly by over a decade near $290 \mathrm{~km}$; the reason for the drop was not clear.

The thermal ion density and temperature measurements from TPA, at different local times and altitudes (above 150 $\mathrm{km}$ ) and in different seasons near solar maximum, will be used to determine the ionospheric scale height at different 
SZA's and the presence or absence of ionopause both at and away from the subsolar point in the topside Martian ionosphere.

The solar EUV flux incident on a planet, and hence the photo-ionization rate in its atmosphere, vary as the inverse square of its distance from the Sun, $R$. On a planet with no intrinsic magnetic field, internal magnetic pressure is not a factor in the overall pressure balance. Consequently, the dayside ionospheric plasma pressure at an altitude of a given neutral density is expected to vary inversely with $R$; this is because the electron density is controlled primarily by its recombination rate and varies as the inverse of $R$, while the electron temperature is governed basically by the spectral shape of the EUV flux and essentially independent of the distance.

Explicitly, assuming photochemical equilibrium in a planetary upper atmosphere dominated by a neutral species, $n$, $P=n_{\mathrm{n}} \int F(v) \sigma(v) d v \propto n_{\mathrm{n}} R^{-2}, L=\alpha n_{\mathrm{e}}^{2}, P=L$, and therefore $n_{\mathrm{e}}=(L / \alpha)^{1 / 2} \propto R^{-1}$ where $P$ is the ion production rate, $L$ is the loss rate, $n_{\mathrm{n}}$ is the neutral density, $F(v)$ and $\sigma(v)$ are the solar EUV intensity and photoionization cross section at frequency $\nu$, and $\alpha$ is the recombination coefficient.

The upper atmospheres of Mars and Venus are very similar. Both are dominated by $\mathrm{CO}_{2}$ and its photochemistry. If Mars has no intrinsic magnetic field as is the case on Venus, the dayside ionospheric plasma pressure on the two planets would be expected to be roughly proportional to the inverse of their respective distance from the Sun; the ram pressure of the solar wind on the two planets would be proportional to the inverse square of the distance. Compared with Venus which has an ionopause, Mars would be more likely to have an ionopause and a topside ionosphere in diffusive equilibrium and characterized by a "diffusive" plasma scale height $H_{\mathrm{i}, \mathrm{d}}\left(=k\left(T_{\mathrm{i}}+T_{\mathrm{e}}\right) / m_{\mathrm{i}} g\right.$ where $m_{\mathrm{i}}$ and $T_{\mathrm{i}}$ are ion mass and temperature, $g$ is the acceleration due to gravity, and $k$ the Boltzmann constant).

If Mars has at least a weak intrinsic magnetic field, the upward diffusion of the dayside ionospheric plasma would be suppressed at low magnetic latitudes where the field is expected to be horizontal, and its topside ionosphere would have a "photochemical" scale height $H_{\mathrm{i}, \mathrm{p}}$ that is twice the neutral scale height $\left(=2 k T_{\mathrm{n}} / m_{\mathrm{n}} g\right.$ where $m_{\mathrm{n}}$ and $T_{\mathrm{n}}$ are the neutral mass and temperature).

On Viking 1 and 2 at $44^{\circ} \mathrm{SZA}$, the measured plasma scale height of $29 \mathrm{~km}$ was comparable to but somewhat larger than twice the neutral $\mathrm{CO}_{2}$ scale height $(\sim 11 \mathrm{~km}$, (Nier and McElroy, 1977)), suggesting that the ionosphere may be closer to photochemical than diffusive equilibrium. The absence of an ionopause in the Martian topside ionosphere and the presence of a photochemical scale height at different local times, if observed, would provide a strong evidence for an intrinsic magnetic field.

The thermal ion measurements will also be used to derive ion drift velocity in the topside Martian ionosphere in both the vertical and horizontal directions. Using a one-dimensional model for the external magnetic field and assuming negligible plasma pressure, Axford (1991) studied the dependence of plasma drift in the subsolar dayside ionosphere on the presence of intrinsic magnetic field in the Martian ionosphere. When the intrinsic field below the ionosphere is smaller than the stagnation field on the topside, the scale height equals the neutral scale height at high altitude and increases to twice the value at low altitude. Also, the plasma drifts downward from the topside. When it has the same magnitude as the external field, photochemical equilibrium prevails and there is no plasma drift. When it is larger than the stagnation field, the scale height can become very large, and the upward plasma drift can exceed the $1 \mathrm{~km} / \mathrm{s}$ level. The precision of velocity estimates from the TPA measurements is estimated to be on the order of $100 \mathrm{~m} / \mathrm{s}$ (in the spacecraft ram component) or better (in the other components), and sufficient to ascertain the possible presence of vertical ion drift and its direction and magnitude.

In the presence of a weak magnetic field, plasma convection may play an important role in the dynamics of the Martian ionosphere since the latter is accessible to the solar wind down to relatively low latitudes. Rassbach et al. (1974) derived a potential drop of $\leq 20-100 \mathrm{~V}$ across the dayside ionosphere, by assuming the merging rate of interplanetary and Martian field lines to be controlled by the ionosphere and a magnetic field of $60 \mathrm{nT}$ at the surface. The distribution of convection electric field on Mars is likely to be dependent on its magnetic field topology, and is currently a matter of theoretical conjecture. The low-latitude orbit of Planet-B precludes ion convection measurements in the polar region where the electric field might be strongest, but at the same time facilitates measurements in the flank region of the Martian magnetosphere where plasma transport is believed to be important.

\section{Solar Wind Interactions}

Because of the weak or non-existent core magnetic field on Mars, the solar wind interacts directly with the upper Martian atmosphere and ionosphere. The interaction results in primarily two types of ion outflows: $\mathrm{keV}$ ion beams from the central tail near the plasma sheet; and lower energy ions from the topside ionosphere, which gradually pick up speed along the flanks and form a mantle between the central tail and the mass-loading boundary.

On Phobos-2, Lundin et al. (1991) observed a transition region across the bow shock near the subsolar point which is indicative of strong braking of the solar wind. The region is characterized by a gradual decrease of solar wind ion velocity outside the bow shock, and an almost complete cessation of flow and a significant increase in heavy Martian ion density inside the bow shock. The direct momentum transfer between the solar wind and heavy Martian ions results in a "loaded" ion pickup process. This process was also observed in the transition region between the magnetopause and the mass-loading boundary in the magnetotail. It effectively extends the topside atmosphere to as far as the bow shock, and results in significant depletion of heavy atmospheric species on cosmogonical time scales. The estimated maximum escape rate of atmospheric oxygen under the assumption of total momentum exchange is very high $\left(\sim 5 \times 10^{26}\right.$ ions s $\left.^{-1}\right)$, and exceeds the oxygen ion escape flux from the terrestrial ionosphere.

The Phobos observations suggest the ionosphere to be the cold plasma source for the observed molecular ions and energetic heavy ion fluxes in the Martian magnetosphere. Mea- 
surements of both the thermal and suprathermal plasma by TPA on both the dayside and the nightside will make it possible to determine the density distribution of the cold plasma population in the nightside ionosphere, and to study the energization path of the energetic ion beams as they evolve from cold ionospheric plasma to energetic ion beams in the magnetotail.

\section{Conclusion and Discussion}

The absence of a strong magnetic field on Mars results in the direct interaction of its upper atmosphere and ionosphere with the solar wind. This interaction results in significant outflows of ions from the Martian ionosphere. The presence of an intrinsic field, however small, is believed to have direct consequences on the structure of the topside ionosphere and its plasma drift velocity distribution. Measurements from Planet-B TPA will be used to study the plasma drifts in the Martian ionosphere and its detailed solar wind interaction.

The question of whether or not Mars has an intrinsic magnetic field remains open. Some of the existing evidences suggest the presence of a weak intrinsic magnetic field (Hanson and Mantas, 1988), while others may be consistent with a purely induced field (Russell, 1986); recent measurements from the Mars Global Surveyor reported in the popular media suggest the presence of localized regions of intense subsurface magnetic anomalies around the Martian planet. At issue is whether the magnetic field that pervades the Martian ionosphere arises from an intrinsic magnetic moment or is induced by the draping of the interplanetary magnetic field. A critical factor in the interaction of an unmagnetized planetary atmosphere with the solar wind is the solar wind stagnation pressure to ionospheric plasma pressure ratio. Slavin and Holzer (1982) suggested that the magnetic pressure from the induced field cannot exceed the ionospheric plasma pressure. They argued that the combination of the two is insufficient to balance the topside solar wind pressure and to divert the solar wind flow around Mars without the presence of an intrinsic magnetic moment. In the lower ionosphere where Pedersen current is available to provide the magnetic pressure bal- ance $(J \times B$ force), the criterion of Slavin and Holzer may not be applicable. However, in the upper ionosphere where Pedersen current is negligible, the partial plasma pressure of suprathermal ions above $15 \mathrm{eV}$ and electrons above $75 \mathrm{eV}$ (energy limit of Viking RPA measurements) may be significant. Particle measurements from TPA up to $200 \mathrm{eV}$ will be used to study the applicability of this criterion.

Acknowledgments. We would like to acknowledge the support from the Canadian Space Agency (CSA) for the Thermal Plasma Analyzer instrument, from the Institute of Space and Astronautical Science (ISAS) for the Planet-B mission, and from the Natural Science and Engineering Research Council (NSERC) under Research Grant OGP0183733.

\section{References}

Axford, W. I., A commentary on our present understanding of the Martian magnetosphere, Planet. Space Sci., 39, 167-173, 1991.

Dolginov, Sh., Comments on "The magnetic field of Mars: Implications from gas dynamic modelling" by C. T. Russell, J. G. Luhmann, J. R. Spreiter, and S. S. Stakara, J. Geophys. Res., 91, 12143-12148, 1986.

Hanson, W. B. and G. P. Mantas, Viking electron temperature measurements: evidence for a magnetic field in the Martian ionosphere, J. Geophys. Res., 93, 7538-7544, 1988.

Hanson, W. B., S. Sanatani, and D. R. Zuccaro, The Martian ionosphere as observed by the Viking retarding potential analyzers, J. Geophys. Res., 82, 4351-4363, 1977.

Lundin, R., E. M. Dubinin, H. Koskinen, O. Norberg, N. Pissarenko, and S. W. Barabasj, On the momentum transfer of the solar wind to the Martian topside ionosphere, Geophys. Res. Lett., 18, 1059-1062, 1991.

Nier, A. O. and M. B. McElroy, Composition and structure of Mars' upper atmosphere results from the neutral mass spectrometers on Viking 1 and 2, J. Geophys. Res., 82, 4341-4349, 1977.

Rassbach, M. E., R. A. Wolf, and R. E. Daniell, Jr., Convection in a Martian magnetosphere, J. Geophys. Res., 79, 1125-1127, 1974.

Russell, C. T., Reply, J. Geophys. Res., 91, 12149, 1986.

Slavin, J. A. and R. E. Holzer, The solar wind interaction with Mars revisited, J. Geophys. Res., 87, 10285, 1982.

Whalen, B. A., D. J. Knudsen, A. W. Yau, et al., The Freja F3C Cold Plasma Analyzer, Sp. Sci. Rev., 70, 541-561, 1994.

Yamamoto, T. and K. Tsuruda, The Planet-B mission, Earth Planets Space, 50, this issue, 175-181, 1998.

A. W. Yau (e-mail: yau@space.phys.ucalgary.ca), G. P. Garbe, M. J. Greffen, W. Miyake, and E. Sagawa 